\title{
LA MEMORIA COMO ACCIÓN SOCIAL A TRAVÉS DE GENERACIONES COETÁNEAS Y CONTEMPORÁNEAS 1938-1998 EN NUEVA CARACAS, CA- TIA (CARACAS, VENEZUELA) ${ }^{1}$
}

\author{
Leilany ESTRADA ${ }^{2}$
}

\begin{abstract}
Resumen
Utilizando una metodología cualitativa longitudinal vinculamos la teoría analítica de las generaciones elaborada por Ortega y Gasset (1923-1939) y la memoria urbana, para reconstruir narrativas basadas en las experiencias de vida de los habitantes de Nueva Caracas (en Catia, Caracas), tomando como referencia la generación precursora, aquella que permitió cambios decisivos en la instalación del sistema de vigencias en Venezuela para 1936.
\end{abstract}

Palabras clave: memoria colectiva, historia local, ciudades urbanas, generaciones, comunidad.

\begin{abstract}
Through the longitudinal qualitative methodology, we link the analytical theory of generations elaborated by Ortega y Gasset (1923-1939) and urban memory, to reconstruct narratives based on the life experiences of Nueva Caracas's settlers (in Catia, Caracas), taking as reference to the precursor generation, the one that made decisive changes in the installation of the Venezuelan validity system since 1936.
\end{abstract}

Keywords: collective memory, local history, urban cities, generations, community.

\section{Résumé}

\footnotetext{
${ }^{1}$ Este artículo es el resultado de una investigación sobre la memoria como acción social a través del estudio de las generaciones de 1938 a 1998 realizada durante el año 2016 en Nueva Caracas, Catia (Caracas, Venezuela) y presentada en Octubre de 2016 para la obtención del título de Socióloga en la Facultad de Ciencias Económicas y Sociales de la Universidad Central de Venezuela. Tutor: Prof. Carlos Viso F. Jurado: Dr. Emilio Osorio Álvarez y Dr. Julio de Freitas Taylor.

${ }^{2}$ Socióloga egresada de la Universidad Central de Venezuela, Adscrita al Laboratorio de Antropología Aplicada de la Facultad Latinoamericana de Ciencias Sociales en Argentina, enfocada en la línea de investigación sobre memoria, estudios urbanos y medio ambiente. Diplomatura en Antropología Social y Política y Candidata a Magíster en Antropología Social por FLACSO, Argentina. Buenos Aires, CP: 1650. Correo electrónico: leilanyestrada@gmail.com
}

Fecha de recepción del artículo: Julio2021

Fecha de evaluación: Septiembre 2021 
À travers la méthodologie qualitative longitudinale, nous lions la théorie analytique des générations élaborée par Ortega y Gasset (1923-1939) et la mémoire urbaine, pour reconstruire des récits basés sur les expériences de vie des colons de Nueva Caracas (Caracas, Venezuela), prenant comme référence génération précurseur, celle qui a apporté des changements décisifs dans l'installation du système de validité vénézuélien depuis 1936. Mots clés: mémoire collective, histoire locale, villes urbaines, générations, communauté.

\section{Introducción}

En este trabajo accedimos a la "ciudad escondida" de José Ignacio Cabrujas, "Nueva Caracas" para los catienses ${ }^{4}$; una ciudad dentro de la Ciudad ubicada al nor-oeste de Caracas (Venezuela). A partir de la teoría y método de José Ortega y $\operatorname{Gasset}^{5}(1957,2005)$, sobre el análisis y construcción de las generaciones históricas y contemporáneas a través del hacer memoria, sostendremos que por medio del uso del lenguaje las personas podemos transferir recuerdos, vivencias, saberes y conocimientos a otros. Esto nos permite lo que menciona Maurice Halbwachs ${ }^{6}(1994,2004)$; no somos seres aislados porque cada memoria que recreamos nos lleva a relacionarnos con los otros, y, gracias a esa memoria y a los cambios y transformaciones personales y colectivas es que somos capaces de visualizar nuestra capacidad creadora, es decir, el otro me acompaña y me ayuda a reconstruir mi experiencia de vida.

Siguiendo este orden de ideas, Ortega y Gasset en "La idea de las generaciones" (lección dictada en 1933), explica cómo las vidas se suceden: mientras unos hombres nacen, otros mueren. En este dinamismo no tenemos control alguno y, por ello, podemos hablar de generaciones contemporáneas y coetáneas. Aclaramos que contemporáneo no es lo mismo que coetáneo, somos contemporáneos en la medida en que compartimos un mismo

\footnotetext{
${ }^{3}$ La "ciudad escondida" es el término utilizado por el escritor venezolano, José Ignacio Cabrujas, nacido en Caracas en 1937, cuya infancia y adolescencia transcurrieron por las calles de Catia. La "ciudad escondida" fue utilizada para describir a Caracas por su autor como "una ilusión de inconformes", "un mientras tanto y por si acaso" que nos hace reflexionar sobre lo efímero que resultan los recuerdos para una ciudad que sufre cambios constantes y, cuyos habitantes, son protagonistas y reflejan sus experiencias de vida sobre las transformaciones que buscan constituir a "Santiago León de Caracas" como una ciudad moderna. Para Cabrujas (1990), Caracas representaba una ciudad indefinida que "conspira contra la memoria", siendo ésta conformada por grandes derrumbadores donde cada intento por descubrir sus espacio terminaría siendo "un fracaso". Mientras revisamos esas notas, surgieron ciertas preguntas; ¿qué había antes "allí"?, ¿qué tipo de vida gestionaba la ciudad antes de sus demoliciones?, ¿qué memorias nos quedan de la "Gran Ciudad" y qué memorias estamos constantemente construyendo?

${ }^{4}$ La palabra "catiense" es una forma cotidiana de llamar al gentilicio de Catia. Asimismo, encontramos que el ser catiense refiere a una persona que vive o ha vivido en cualquier sector de la parroquia Sucre (Nueva Caracas, Propatria, La Silsa, El Cuartel, entre otras localidades). Sería la forma en que las personas oriundas de la zona nor-oeste de Caracas son denominadas y auto-denominadas coloquialmente. También una forma de identificarse y adscribirse al lugar: es común escuchar "yo soy de Catia" o "soy catiense".

${ }^{5}$ Véase: Ortega y Gasset, J. (1957): El hombre y la gente. Obras inéditas. Madrid: Revista de Occidente; Ortega y Gasset, J. (2005): En torno a Galileo. Madrid: Biblioteca Nueva y Marías, J. (1989): Generaciones y constelaciones. Madrid: Alianza editorial, S.A.

${ }^{6}$ Véase Halbwachs, Maurice (2004): Los Marcos Sociales de la Memoria. Título original: Les cadres sociaux de la mémoire (1994). París: Albin Michel. Coedición: Facultad de Ciencias Sociales de la Universidad de Concepción en Chile y Facultad de Ciencias Económicas y Sociales de la Universidad Central de Venezuela. Caracas: Anthropos.
} 
espacio temporal y físico ${ }^{7}$, pero incidimos en la historia de manera distinta haciendo posible la innovación humana, lo que sería en términos de Ortega y Gasset: "el anacronismo esencial de la historia", que define a cada generación como "el conjunto de los que son coetáneos y contemporáneos en un actual círculo de convivencia” (Marías 1989: pp 7).

Esto último es clave para acercarnos a la idea de las generaciones. Tal como explica Marías (1989: pp. 31), podemos presenciar este "anacronismo esencial de la historia", donde todos convivimos, experimentando e interpretando las vivencias desde nuestras propias circunstancias de vida por medio de la edad, ya que la edad en términos del autor mencionado es "el modo que tiene el tiempo de quedar en nosotros" (Idem.). En nuestro caso, lo vemos reflejado dentro de los hitos que sufrió la ocupación, apropiación y uso del espacio en Nueva Caracas, es decir, nos ubica en un antes y un después de la constitución de la urbanización Nueva Caracas en Catia (aproximadamente 1936). Vale aclarar que la década 1930-1940 no es tomada de manera arbitraria, sino más bien, va pensada en que cercano a esta fecha se comienza a visualizar la planificación y modernización de las zonas populares de Catia debido a su expansión poblacional y a la función que desempeñó respecto al resto de la ciudad de Caracas, así como también, los lugares autoconstruidos que constituyen los barrios populares de Caracas.

Por lo tanto, si entrecruzamos la memoria urbana con la idea de las generaciones encontramos los significados y el valor que puede tener para una localidad el acceder a los recuerdos de transformaciones y cambios en el espacio urbano. Por lo tanto, el acceso a la memoria de Nueva Caracas acompañada con la historia de Caracas, y la propia historia de los venezolanos, nos permite acercarnos a sus generaciones históricas, para finalmente, conocer la memoria colectiva, las relaciones sociales, la pertenencia, los encuentros y los des-encuentros.

Primeramente, comenzaremos por mencionar la historia local de la urbanización Nueva Caracas; luego, explicaremos las perspectivas utilizadas para el abordaje teórico de la memoria como acción social en esa localidad para adentrarnos en la reflexión sobre la teoría analítica de las generaciones y metódica de la historia planteada por Ortega y Gasset (1957) y continuado por Julián Marías (1989) y, de esta manera, utilizando una metodología cualitativa longitudinal vinculamos la memoria urbana y las generaciones coetáneas y contemporáneas de Nueva Caracas, tomando como representante de ésta, la generación precursora previamente identificada y sustentada por Carlos Viso (2012) aquella que acompañó cambios decisivos en el curso y continuidad histórica de aquél sistema de vigencias instalado en Venezuela desde 1936.

\section{Contexto histórico de Catia: una ciudad dentro de una Ciudad}

Catia es el lugar geográfico donde se encuentra Nueva Caracas. Esta zona inicialmente fue un lugar de comunicación e intercambio comercial desde la gran ciudad hacia el Puerto de La Guaira en el Estado Vargas. Durante siglos significó una de las principales salidas naturales, convirtiéndose en uno de los destinos predilectos para dirigirse hacia otras partes del país. Vale destacar que previo a la expansión que conocemos ahora, el

\footnotetext{
${ }^{7}$ La etimología de la palabra contemporáneo viene del prefijo con- (a la par), tempus (tiempo) y el sufijo aneo (pertenencia) a la par con el tiempo. Mientras que la palabra coetáneo viene del latín coaetaneus, co(encuentro) y aetas (contracción de aevitas, que nos da edad) lo que sería "de la misma edad".
} 
camino llegó a estar cerrado por representar una amenaza ya que el acceso hacia la costa atraía filibusteros (piratas y corsarios) (Antillano, 1999). Así fue como se habilitó otra entrada y salida de la costa hacia la ciudad (Puerta Caracas en La Pastora). Este hecho, hizo que Catia estuviese aislada durante un tiempo (desde S.XVII hacia el S.XIX aproximadamente). Una vez recuperado su camino natural, éste se convierte en un puente por donde es construida la primera carretera de la República ("La Carretera de la Guaira" de 1845) y, es en ese momento, cuando Catia comienza a tomar aspecto de barriada urbana.

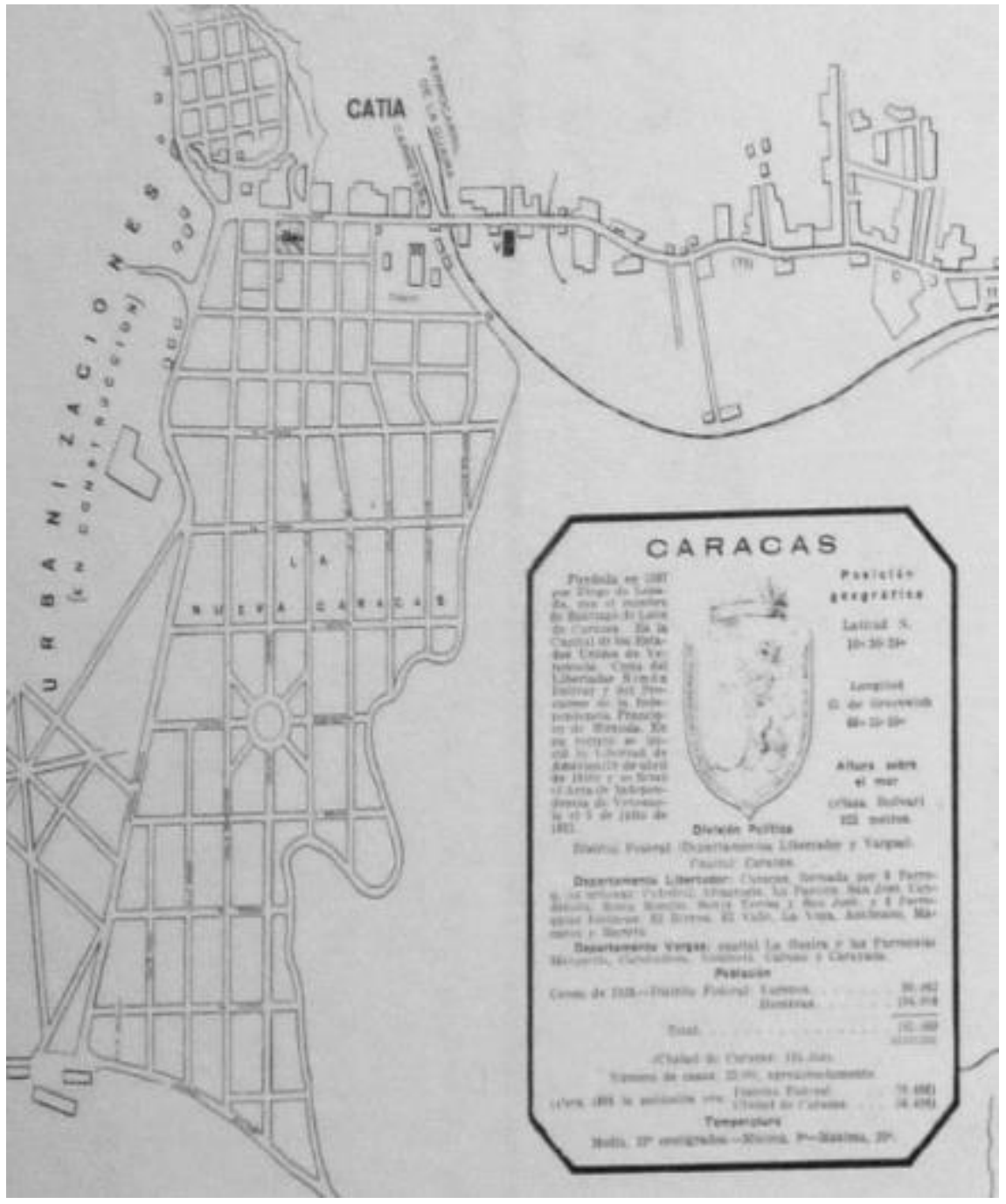

Fuente: Plano No. 68 de "Contribución al estudio de los planos de Caracas” de Irma De Sola Ricardo.

Acercándonos al periodo que nos concierne, en 1930 Venezuela iniciaba la década que marcaría el fin de la dictadura gomecista (1908-1935), mientras se acoplaban a los cambios innovantes que surgían en las zonas más urbanas de Caracas, los pocos espacios rurales que iban quedando sugerían la creación de nuevos espacios habitacionales, siendo este hecho muy importante para la integración socio-urbana de Catia con la Ciudad. Cabe destacar que Catia está ubicada en una tierra accidentada con ríos, quebradas y lagunas que la rodean, además de componer un espacio denso poblacionalmente. De hecho, tal

\footnotetext{
${ }^{8}$ Actualmente sigue habilitado este camino pero es llamado como la "carretera vieja Caracas-La Guaira" rodeada por diferentes barrios populares y quebradas.
} 
como recuerdan las primeras generaciones, existió una icónica y extensa laguna llamada "La Laguna de Catia" cuya eliminación coincide con la creación urbanística del sector Nueva Caracas tal como hoy se conoce. En términos generales, las zonas que componen Catia, gozan de distintas características positivas; principalmente su proximidad con $\mathrm{Ca}$ racas y con el puerto de la Guaira, además de un clima cálido y fresco.

La inauguración de Nueva Caracas se dio aproximadamente durante el gobierno del General Eleazar López Contreras (1936-1941). No obstante, ante la expansión de Caracas y la planificación de sectores cada vez más enriquecidos, Catia, en general, se volvió una zona marginada pensada como el "patio trasero de Caracas" y, volviéndose también, un "sector combativo" donde se erigieron diferentes luchas sociales con el fin de solicitar atención ante la recurrente despreocupación de las autoridades gubernamentales, el abandono y la poca incidencia en políticas públicas.

A partir de 1940 Catia intensifica su industria comercial y, junto al crecimiento demográfico, las nuevas migraciones campo-ciudad como consecuencia de la renta petrolera y, posteriormente, la administración -dictatorial- de Marcos Pérez Jiménez (1952-1958), impulsaron la transformación de todos los sectores de Caracas. Durante esas décadas los proyectos de infraestructura urbana eran parte del proyecto de modernización de la Ciudad y, es finalmente cuando se erige la autopista Caracas-La Guaira (1953), reemplazando la antigua carretera.

Particularmente, dentro de esa época se tuvo la oportunidad de conocer el relato vivo de una mujer nacida en 1933, Celina Fehr ${ }^{10}$ quien nos dice con detalle qué era lo más significativo en sus memorias: "Los carnavales en Catia eran una belleza, eran indescriptibles. Recuerdo que estábamos en los tiempos de Pérez Jiménez, y, a pesar de ese clima dictatorial, podías ir a la plaza Catia, confeccionabamos los disfraces y disfrutabamos". Asimismo, empalma sus recuerdos sobre los gobiernos que formaron parte de la vida de los catienses y de los venezolanos, para hablarnos de esos períodos dictatoriales:

Yo vi el principio del gobierno de López Contreras siendo pequeñita, el gobierno de Medina y viví todo el gobierno de Pérez Jiménez. Todas eran dictaduras, pero eran diferentes aunque ibas a votar, ellos salían ganando siempre. La de Gómez que fue horrible, después vino la de López Contreras que hubo un poco más de democracia, un respiro y luego Pérez Jiménez, ese hombre si era malo (Celina Fehr) ${ }^{11}$.

\footnotetext{
${ }^{9}$ La Laguna de Catia fue drenada por el Banco Obrero (BO), siendo ésta un fenómeno natural de la quebrada Caroata y según data desde 1557. Para 1934 según Eduardo Röhl tendría una superficie de $35.100 \mathrm{~m} 2$ y una profundidad entre 10 y 12 metros. Según la Fundación Arquitectura y Ciudad la Laguna fue drenada en su totalidad en 1940 para ampliar las avenidas de la nueva urbanización.

${ }^{10}$ A partir de la mención de Celina Fehr iremos introduciendo los relatos recolectados en las entrevistas para presentar el cuadro que explica la metodología de este trabajo investigativo, el cual ubica en qué generación coetánea y contemporánea se encuentran nuestros interlocutores. En el caso de Celina Fehr, nuestra interlocutora de 83 años para el 2016, está ubicada en la "Generación C" perteneciente a la Zona de Fechas 1931-1945. Los que para 1968 cumplirían los 30 años.

${ }^{11}$ Este testimonio es parte de las memorias compartidas de Celina Fehr sobre su vida en Catia durante nuestra primera entrevista realizada el 14 de Junio de 2016. Con Celina hicimos dos entrevistas, además, nos llevó a recorrer el histórico bar ubicado en Nueva Caracas, "El Torero", mientras nos presentaba al "Sr. Peter" (el dueño del bar) y nos indicaba sobre la colección de cosas con historia guardadas en esa casa colonial ubicada en la calle Maury de la parroquia Sucre. Véase nota: "Iconos de Caracas: El Torero, una ventana al pasado". Publicado el 19 de agosto de 2013 por “Tu Zona Caracas". Disponible en: https://www.youtube.com/watch?v=D1fVCC7zMGQ
} 
Esto nos recuerda al semiólogo Armando Silva, cuando describe cómo podemos construir y visualizar una ciudad creada desde nuestro imaginario, es decir, una ciudad subjetiva que nos lleva a tener afectos hacia la misma. Por ello nos preguntamos; ¿qué recuerdos se alojan en la memoria urbana de Nueva Caracas? Silva (1994) escribe;

La ciudad puede proyectarse como un cuerpo humano, con sexo, corazón, miembros, pero también con sentidos: huele, sabe, mira, oye y se hace oír y se interviene colectivamente. La ciudad, así corresponde a una organización cultural de un espacio físico, mediático y social (Silva 1994: 400).

La memoria al ser relacional y compartida nos permite comprender que no existe ningún recuerdo que no sea social. Y, siguiendo la afirmación de José Ignacio Cabrujas, "Caracas es una ciudad donde no puede existir ningún recuerdo. Es una ciudad en permanente demolición que conspira contra cualquier memoria" (Cabrujas en Socorro, 1994: 54), nos interesa ver cómo las personas recrean sus memorias urbanas sobre Nueva Caracas para construir y (re)encontrarse con el sinfín de relaciones sociales que el ejercicio de recordar (real o imaginariamente) trae. Por esa razón, cuando Celina Fehr comenta acerca de su devoción cristiana nos cuenta como Catia se transformó en el lugar donde junto con otros vecinos y generando nuevos lazos, construyeron una iglesia luterana capaz de expandir tales relaciones, convirtiendo la zona en un punto de referencia para los luteranos: “[...]ahí me casé, a los 26, casi cumpliendo los 27 años. Mi esposo y yo teníamos la misma edad. Nos casamos en la iglesia que junto con la comunidad pudimos construir" (Celina Fehr).

\section{Referencias conceptuales: La memoria como acción social}

Respondiendo a nuestra pregunta central: ¿cómo la teoría analítica de las generaciones y su método histórico contribuyen en la reconstrucción de la memoria colectiva y generacional de Nueva Caracas? y, ¿cómo vinculamos la memoria como acción social y generaciones? nos vemos en la obligación de explicar algunos aspectos centrales sobre el estudio de la memoria, por ejemplo; las diferencias que tiene con la historia. Vázquez nos recuerda que ' 'la memoria se refiere al pasado, pero no es el pasado; de la misma forma que el pasado, tampoco es la historia"' (Vázquez 2001: 53). Por lo tanto, es necesario distinguir y deslindar lo que significa la reconstrucción de la memoria social de Nueva Caracas sin confundirla con la reconstrucción historiográfica del pasado, que tiene sus enfoques y métodos de acuerdo a sus pretensiones de cientificidad.

En este sentido, cuando hablamos con otra de nuestras interlocutoras, Edith Torres ${ }^{12}$ (vecina de Catia nacida en 1953) sobre cómo era Nueva Caracas en la época que transcurrió su adolescencia. Pudo recordar que aproximadamente para 1968;

[...] era una época en la que todo se mantenía y transcurría en Catia; las fábricas, las empresas, las industrias. No tenías necesidad de irte a otro lugar. Desde acá se exportaban materiales para otras partes de Caracas y Catia crecía junto a la ciudad, mientras ésta se desplegaba hacia otras zonas (Edith Torres)

Esto último nos recuerda a Halbwachs (1968, 2004), cuando escribe que las personas al momento de contar y desarrollar su memoria colectiva, no colocan líneas de separación

\footnotetext{
${ }^{12}$ Edith pertenece a la generación D., la que sigue después de Celina, es decir, a la zona de fechas o nacidos entre 1946-1960; aquellos que para 1983 tendrían 30 años. Edith Torres hace memoria desde 1968, cuando ella tendría aproximadamente 15 años.
} 
trazadas como en la historia, en su lugar, aparecen límites irregulares e inciertos pues no podemos comprobar si esas memorias son certeras aunque sí podemos conocer una cronología de hechos históricos de la ciudad. Para Edith, si bien Catia en un principio era una zona industrial actualmente:

[...]creció y se dispersó. La gente empezó a trabajar cada vez más lejos porque ya no había fuentes de trabajo acá, teniendo el metro cerca la conexión con Caracas era cada vez más cercana y también era accesible llegar a otros lugares. Los tratos vecinales ya no son familiares porque cada vez somos más personas, hay cada vez más desconocimiento (Edith Torres).

Estas palabras expresadas desde la memoria no están escritas en libros de historia local, es posible que someramente, se encuentre algo relacionado a la industria pero jamás encontraremos la expresión del sentir que Catia "ya no es una zona familiar" tal como lo expresó Edith.

Por ello, para trabajar con los conceptos de memoria tenemos en cuenta que muchas veces el problema radica en la recurrente intención de transformar la memoria en historia, siendo ésta última una ciencia social y la memoria un patrimonio privado, ubicada al interior del acontecimiento y, que por supuesto, toma como referencia no sólo los relatos de sí misma sino también los que surgen a su alrededor: historia, acontecimientos, hechos, textos, entre otros, provocando de esta forma una "relación de ósmosis" entre historia y memoria (Vázquez, 2001), siendo esto último, reflejado entre nuestros interlocutores.

En otras instancias, la memoria social también nos permite acceder a la capacidad de conmovernos e identificarnos cuando alguien nos cuenta una historia. Junto a Vázquez (2001) nos preguntamos ¿cómo ese patrimonio interior y genuino que tenemos las personas puede llegar a resultarnos -simultáneamente- tan familiar, inteligible y común? La clave radica en un uso social por excelencia: el lenguaje. Cuando Nueva Caracas a través de sus vecinos habla, $y$, se comunica, permite que la memoria social se vuelva acción comunicativa.

Sin el lenguaje no podría haber memoria compartida y es a través del lenguaje cómo, por ejemplo, otro interlocutor llamado Eduardo Flores ${ }^{13}$ (nacido en 1985) nos cuenta cómo siendo un niño pudo experimentar diferentes acontecimientos político-económicos que lo llevaron a vivir emociones fuertes sobre la historia venezolana;

[...] ]en 1992 ocurrieron algunos acontecimientos que me marcaron, viví los intentos de golpe de Estado al gobierno de Carlos Andrés Pérez en Caracas. Yo vivía con mi familia en los Frailes de Catia y por primera vez en Venezuela un avión rompió la barrera del sonido. Ese día quedamos tumbados en el piso con zumbidos en los oídos y vidrios por todos lados. Es algo que jamás olvidaré (Eduardo Flores).

Este relato muestra que cuando contamos lo que recordamos existe una implicada relación con los otros a través del lenguaje, proveedor de sentido y significados de las interpretaciones que hacemos. Tal como escribe Halbwachs "cuando el hombre cree encontrarse solo, cara a cara consigo mismo, otros hombres emergen y, con ellos, los grupos de los cuales proceden" (Vázquez 2001: 79) en tanto que la función primaria del lenguaje será la "construcción de mundos humanos" (2001: 86).

\footnotetext{
${ }^{13}$ Eduardo Flores perteneciente a la generación $\mathrm{F}$ dentro del esquema que elaboramos, y, al momento de contarnos sobre Catia tenía 31 años. Las memorias que nos trae son las pertenecientes a su yo de 7 años.
} 
Finalmente, es importante señalar que hacemos memoria desde el presente, una especie de reconstrucción que implica interpretaciones de las trayectorias hechas o acontecidas, y de las posibles o futuras. Vázquez afirma: 'Todos los futuros arrancan del presente ya que este es el topos y el cronos de la posibilidad" (2001: 103). En el presente también tenemos al pasado; cada vez que contamos algo, cada vez que conocemos a alguien, nuestra memoria bajo la posibilidad de selección, se dirigirá y contará lo que le parezca oportuno recordar, de acuerdo a los intereses que se tengan en el presente. Por lo tanto, la memoria es circunstancial. Lo que nos lleva a interpretar lo dicho por Eduardo Flores sobre cómo cree que la localidad va cambiando transmitiendo su sentir, en el que la realidad catiense sufrió cambios:

[...] a veces uno no necesita que te digan las cosas o verlas, no se trata tanto de emitir una opinión, basta con percibir lo que transmite la ciudad. Y yo que conocí a Catia en un momento, ahora, su aroma... su sentir... no es lo mismo que antes (Eduardo Flores).

\section{Teoría analítica de las generaciones y metódica de la historia}

En 1949 se publicó "El método histórico de las generaciones” de Julián Marías ${ }^{14}$, un libro que sistematiza los fundamentos filosóficos de la teoría analítica de las generaciones elaborado por Ortega y Gasset desde 1923 hasta 1939. Ese texto nos permitió cuestionar: ¿qué son las generaciones? ¿cuánto duran? ¿cómo podemos determinarlas en Nueva Caracas?

Intencionalmente hemos ido introduciendo parte de los relatos que nuestros interlocutores compartieron para visualizar cómo las generaciones se constituyen como el órgano visual de la realidad histórica, o como lo dice el propio Ortega y Gasset 'las generaciones son una y misma cosa con la estructura de la vida humana'" (Marías 1989: 111). Desde este lugar, decimos que es posible entender algo humano cuando se cuenta una historia, es decir, aquello que una persona hizo antes, en vista de su circunstancia.

Para sintetizar el método histórico con la teoría analítica de las generaciones elaborada por José Ortega y Gasset utilizaremos la metáfora de la navegación que menciona Marías (1989): El ser humano al no ser un ente aislado, encuentra que la vida es una realidad radical, se trata sin más, de una "razón para navegar", siendo la vida "un elemento móvil, fluido[...]" (1989: 157), adaptarnos a esa movilidad nos permitirá precisamente encontrar seguridad tal como "el barco se ajusta a la fluctuación de las olas" (1989: 158). Esta metáfora ilustra el método de la razón histórica como método de la teoría de las generaciones, lo que también significa una crítica a la precaria comprensión de la filosofía de la razón histórica y vital expuesta por Ortega y Gasset. Es tal la incomprensión señalada que provoca "'...$]$ mareo a los hombres de tierra adentro, que nunca han salido de los carriles de la razón abstracta, buenos para caminar sobre el rígido lomo de las cosas inertes." (1989: 158).

\footnotetext{
14،El método histórico de las generaciones" es el título del libro editado en 1949 producto del curso dictado por Julián Marías en 1948. Para 1989 reeditó el libro bajo el nombre de "Generaciones y constelaciones" donde reproduce el texto de 1949 además de varios ensayos anexados por el autor que ilustran la aplicación del método histórico de las generaciones y su enfoque analítico. Cabe destacar, que Julián Marías fue discípulo de José Ortega y Gasset.
} 
En resumen, la estructura de la vida humana tamizada por la "edad social", es cualificadora del tiempo. Marías lo condensa muy bien cuando expresa: "La edad es el modo que tiene el tiempo de quedar en nosotros" (1989: 31). Señalar que la vida humana está cualificada por la "edad social", implica tomarla en cuenta como un tamiz que permite escoger, esa "edad social" como criterio para identificar las generaciones, y particularmente, para situar a los coetáneos y contemporáneos que habitan el mismo tiempo presente pero con edades biológicas diferentes.

En este contexto, Marías explica un concepto clave en el enfoque de las generaciones: las vigencias, es decir, el sistema de usos sociales vigentes en la sociedad. El sistema de vigencias define el mundo, la circunstancia en la que cada uno tiene que hacer su vida. Esto último nos recuerda cuando Edith Torres nos contó sobre su proceso de formación anclado a los acontecimientos que ocurrían en 1968: para Edith de 15 años "la educación era algo 'impuesto', se trataba de un sistema que ya estaba vigente", y, añade: "fue muy bonito porque se trataba del momento en el que ocurrían cosas en el mundo. En ese momento ocurrieron transformaciones políticas que para mí fueron significativos momentos de cambio" (Edith Torres).

La edad social de las generaciones que señala Marías nos dice que la vida humana puede ser considerada en cinco (5) períodos que permiten distinguir a los que son coetáneos, es decir, aquellos que tienen la misma edad social, determinada por una "zona de fechas". De esta forma, realizamos nuestro análisis para el caso que nos ocupa:

i. 0-15 años - Infancia: Nos encontramos con el mundo ya creado.

ii. 15-30 años - Formación: Etapa en la que nos formamos cómo seres en sociedad bajo ese mundo ya establecido.

iii. 30-45 años - Iniciación: Frente a las incomodidades del mundo que nos recibió, el hombre comienza a actuar, intenta modificar el sistema de vigencias actual para imponer su propia innovación. Es una época en la que se gestan ideas y se lucha con la generación que rige el sistema para intentar desplazarla de su poderío.

iv. 45-60 años - Predominio: Esta generación ha logrado imponerse y es vigente en el mundo ya que logró innovar la edad anterior. Son los que 'están en el poder" y los que intentan luchar para defender lo que construyeron de la generación que se alza.

v. 60-75 años - Vejez ${ }^{15}$ :La época de supervivencia histórica, ya no luchan pero aportan sus experiencias.

Por lo tanto, "la edad [...] no es una fecha, sino una zona de fechas y tienen la misma edad, vital e históricamente, no solo los que nacen en un mismo año, sino los que nacen dentro de una zona de fechas" (Marías 1989: 108). Esto es crucial para comprender la teoría analítica de las generaciones ya que implica identificar dos fases en la etapa de plena eficacia histórica comprendida entre los 30 y 60 años de edad. Es decir;

[...]la de los hombres de treinta a cuarenta y cinco años (gestación) y la de los hombres de cuarenta y cinco a sesenta (gestión). Estos viven instalados en el mundo que han hecho, mientras que los más jóvenes están haciendo su mundo, el que todavía no es vigente (Marías 1989: 109).

\footnotetext{
${ }^{15}$ Hay que destacar que los cambios en la longevidad podrían variar según la esperanza de vida. Pero en general, podemos ubicarnos, tanto a nosotros mismos dentro de las edades sociales e incluso a los que gobiernan para ver cómo se va moviendo el tamiz de las generaciones.
} 
Se trata de dos estructuras de la vida humana diferentes y en constante polémica. Dos generaciones con eficacia histórica (desde los 30 y los 60 años). Son contemporáneas pero no coetáneas. Ante esto, Ortega y Gasset señala que "lo decisivo en la idea de las generaciones no es que se suceden, sino que se solapan o se empalman. Siempre hay dos generaciones actuando al mismo tiempo, [...]con distinto índice de edad y, por ello, con distinto sentido" (Marías 1989: 109). De hecho, analizando las generaciones de Nueva Caracas, encontramos que mientras Ernesto La Cruz ${ }^{16}$ (nuestro interlocutor de 20 años), se encuentra con el mundo ya construído, aprende de él y de su sistema de vigencias actual, mientras que por su lado Omar Pescoso ${ }^{17}$ de 43 años nos recibe en su despacho de contaduría, espacio de trabajo propio y construido desde sus cimientos por él mismo, lo que muestra que dentro del rango de edades de las generaciones con eficacia histórica el se encuentra "imponiendo" su propia innovación.

No obstante, es pertinente considerar lo que se distingue como una "crisis histórica" cuando acontece un tipo de cambio al perder vigencia un antiguo sistema de convicciones, entonces emerge y tiene un papel relevante una generación decisiva, es decir, aquella que

[...]por primera vez piensa los nuevos pensamientos con plena claridad y completa posesión de su sentido: una generación, pues, que ni es todavía precursora, ni es ya continuadora (Marías 1989: 109).

Esto último nos recuerda lo que nos cuenta Omar Pescoso cuando habla de la generación de sus padres y cómo tomaron la decisión de migrar a Venezuela desde las Islas Canarias $^{18}$. El padre de Omar en 1954 con sólo 14 años emigró lejos hacia un país latinoamericano:

[...]él ya conocía a mi mamá desde niño, así que en una oportunidad se volvió a ir a las Islas Canarias y regresó con ella, instalándose desde Catia en 1959. Acá iniciaron vendiendo huevos frescos desde las 6 a.m. hasta muy tarde. Mi papá se iba por toda la plaza Peréz Bonalde ofreciendo los huevos con un cajón y a todo pulmón (Omar Pescoso).

Tener la oportunidad de ver una época a través de esta dinámica (es decir, generacional) significa ver la historia (los hechos y acontecimientos) desde adentro, donde las generaciones se solapan, y cualifican con su edad social el tiempo histórico y social. Pero nos faltaría explicar; ¿cómo determinamos estas generaciones? y, ¿cuál es la zona de fechasedades a la que pertenecen? es decir, cómo es el método. José Ortega y Gasset (1993, 1959, 2005) y Julián Marías (1989) explican que se debe tomar un momento histórico en el que ocurriese un gran cambio o de significado en la vida humana ${ }^{19}$. Seleccionar un

\footnotetext{
${ }^{16}$ Ernesto La Cruz nació en 1996 y representa la generación G.

${ }^{17}$ Omar Pescoso nació en 1973 y para este estudio perteneciente a la zona de fechas de la generación E

${ }^{18}$ Omar Pescoso retrocede a la época en la que sus padres tenían 14 años, y nos cuenta la historia de cómo siendo migrantes nacidos en 1940 se instalaron en Nueva Caracas desde 1954.

${ }^{19}$ Ortega y Gasset, y Julián Marías en "En torno a Galileo" (1933) toman como epónimo en su explicación la época de Descartes con el fin de ilustrar y demostrar la metódica que permite identificar la generación de Descartes. "Tómese un gran ámbito histórico dentro del cual se ha producido un cambio en el vivir humano que es radical, evidente, incuestionable." Y para esto Ortega sitúa las fechas que integran la Edad Moderna, la época de Descartes, y prosigue señalando: En ella vive una generación que por vez primera piensa los nuevos pensamientos con plena claridad y completa posesión de su sentido: una generación, pues, que ni es todavía precursora, ni es ya continuadora. A esa generación llamo generación decisiva. [...] Para esto se busca la figura que con mayor evidencia represente los caracteres sustantivos del período. En
} 
representante para una generación decisiva implica lo siguiente; tomar a alguien que marca un hito en la historia no necesariamente significa que la estructura de la vida de una sociedad esté condicionada por esa persona, más bien, es a la inversa, se trata de la Generación a la que esa persona pertenece.

Agregamos que no se trata en ningún caso de un parecido entre hombres, sino del mundo en que esos hombres vivían, una época que marca un ritmo particular, es decir, "de las vigencias que constituían el mundo de cada uno de ellos" (Marías 1989: 164), por ejemplo, cuando Eduardo Flores nos cuenta sobre los acontecimientos políticos ocurridos en Venezuela durante el año 1999, un nuevo período en la historia venezolana comenzaba a escribirse y, fácilmente podemos identificar cuál era el sistema de vigencias que comenzaba a instalarse en ese momento, el cuál sigue aún vigente:

[...]mi papá me cargó en los hombros y nos fuimos por el medio de la gente y el presidente, en aquel momento, candidato Hugo Chávez, me tocó la mano y yo pensé que me iba a morir. Grité de la emoción. Sentía que estaba tocando a superman (Eduardo Flores).

A continuación presentaremos cómo se analiza la memoria de Nueva Caracas a través de sus generaciones coetáneas y contemporáneas.

\section{Metodología: Memorias de Nueva Caracas a través de sus generaciones}

Los criterios y fuentes que nos permitieron identificar la serie de las generaciones históricas en Venezuela y que nos sirven para visualizar las generaciones en Nueva Caracas, proviene de la línea de investigación cualitativa longitudinal que realiza Carlos Viso $(2012)^{20}$ donde identifica la generación precursora y decisiva de 1938, es decir, los nacidos en la "zona de fechas" 1901-1915, y que tiene como fecha central 1908. Para 1938, esta generación estaría cumpliendo 30 años permitiendo de esta forma lo que explica Ortega y Gasset; el momento en que una generación comienza a tener "eficacia histórica". Esos 30 años marcan ciertas pautas claves de la historia venezolana; tres años después de la muerte de Juan Vicente Gómez, y desde 1936 cuando Eleazar López Contreras (18831973) preside el gobierno hasta 1941. El grupo o representantes de otras generaciones, que por su ubicación o proximidad, y las relaciones con representantes de la generación posterior o anterior, es lo que Marías identifica como "constelación" de una generación.

La infancia y juventud de la generación del 38 transcurre entre los gobiernos de Cipriano Castro 1899-1908, y los presididos directa e indirectamente por Juan Vicente Gómez, específicamente a partir de 1914 cuando se despliega plenamente el carácter dictatorial del gomecismo hasta 1935. Parte del período de formación (15-30 años de edad) de esta generación se ve afectada por las características de este régimen. En ese contexto, la generación del 38 tiene un desempeño que reúne características relevantes de una generación precursora y decisiva en las trayectorias que se abren en el país a partir de 1936,

nuestro caso, no parece discutible que ese hombre es Descartes... Con esto tenemos el epónimo de la generación decisiva, logrado lo cual, el resto es obra del automatismo matemático (Citado por Julián Marías, 1989, pp.162).

${ }^{20}$ Sociólogo y profesor de la Escuela de Educación y de Sociología, de la Universidad Central de Venezuela. Carlos Viso, nos puso al alcance diversos materiales, entre ellos el trabajo inédito (2012): "Cultivador de humanidad entre la niebla: Rafael Augusto Vegas Sánchez (1908-1973)”. Fue junto a él, y tras muchos meses de estudio, conversaciones e intercambios que pudimos analizar las voces de las generaciones de Nueva Caracas, en Catia. 
coincidiendo con esos "30 años", es decir, cuando se inicia la fase de gestación y gestión hasta los 45 años de edad.

Es oportuno señalar que las generaciones; 1953 (nacidos entre 1916-1930 con fecha central 1923) y 1968 (nacidos entre 1931-1945 con fecha central 1938), y tal como nuestra interlocutora Celina Fehr (1933) demuestra en su testimonio, comparten como generación decisiva y continuadora los cambios establecidos a partir del Programa de Febrero ${ }^{21}$ de 1936, y el Plan Político administrativo ${ }^{22}$ de 1939-1941, momentos que marcaron un proceso irreversible de institucionalización en el ámbito social y económico, y evidencian la intervención económica y social del Estado. A la par, la renta petrolera en Venezuela contribuirá en la definición de las relaciones entre ciudadanos, Estado y petróleo, lo que marca cambios significativos en el sistema de vigencias de los usos sociales en el país, y en el que juegan un papel relevante las generaciones del 1938, 1953 y 1968 (Viso, 2012). Por lo tanto, la generación a la que pertenece Celina Fehr será una de las generaciones decisivas para el curso del país hasta el presente.

Con esta explicación, y teniendo en mente la visualización de las generaciones 1901-1928 en Venezuela, buscamos "bajar a tierra" cómo las generaciones conviven entre ellas. Lo significativo de la misma, es que cualquier persona nacida en Venezuela, podría ubicarse e identificarse en alguna de las generaciones a partir de la generación precursora de 1938. Además, en este trabajo hicimos uso de una metodología cualitativa longitudinal que nos permitió desarrollar y sistematizar las etapas desarrolladas. En el diseño longitudinal y el análisis de las generaciones es necesaria la aplicación de técnicas de corte cualitativo, tales como; entrevistas a profundidad, historias orales o historias de vida. Es por ello que realizamos entrevistas semiestructuradas, orientadas a conocer la historia oral de los informantes seleccionados. En total sumamos una muestra de 22 personas vinculadas a Nueva Caracas durante más de cinco (5) meses de etnografía, incluyendo una (1) entrevista a experto, una (1) autobiografía, (3) testimonios orales realizados por otra autora ${ }^{23}$ y quince (15) entrevistas con informantes pertenecientes a las generaciones coetáneas y contemporáneas en 2016.

Asimismo, ayudamos a los interlocutores a visualizar sus lugares de residencia y convivencia en un croquis/mapa de la parroquia Sucre, con sus respectivas delimitaciones. Respecto a la selección de informantes, se comenzó a preguntar a los entrevistados si éstos conocían a otros vecinos que pudiesen dar testimonio de sus vivencias en Catia, siendo el

\footnotetext{
${ }^{21}$ El Programa de Febrero fue un conjunto de medidas anunciadas el 21 de febrero de 1936 por Eleazar López Contreras donde fijó cuáles serían las directrices que tomaría su gobierno y el advenimiento de un cambio político, social, económico y cultural en Venezuela. Tras esas medidas se pudo volver a encarar el nacimiento de una nueva democracia donde los derechos y libertades eran reconocidas dentro de un sistema económico capitalista, reafirmandose garantías constitucionales (sufragio universal), se modernizó el sistema de salud donde se crearon instituciones sanitarias y de protección social, se creó un sistema nacional de carreteras modernas. Respecto a la educación nacional, se pensò en edificar escuelas y en el acondicionamiento de aulas. Luego de largos períodos de economía sustentada dentro de la renta petrolera por primera vez se pensó en sustentar la economía en otros recursos tales como la agricultura y cría, entre otras.

${ }^{22}$ El plan administrativo presentado por López Contreras fue aprobado por el Congreso en 1938 donde se pudiese ampliar y dar continuidad al Programa de Febrero de 1936.

${ }^{23}$ La profesora venezolana Milagros Socorro publicó en 1994 “Catia Tres Voces” y, a través de las historias de vida de María Carrasquel Rivas, Enrique Hernández Mujica y José Ignacio Cabrujas pudimos junto a Celina Ferh y sus memorias analizar la generación precursora del 38.
} 
método snowball el medio por el cual visualizamos cómo la memoria es capaz de relacionar al otro según sus circunstancias y trayectorias.

De las 5 generaciones seleccionadas para este estudio centrado en Nueva Caracas, la primera $(C)^{24}$ de ellas, es decir, la nacida entre 1931-1945 va representada por 4 personas, 3 de ellas ya fallecidas, cuyos testimonios fueron escritos por Milagros Socorro y 1 de ellas, Celina Fehr (1933), cuyo testimonio fue recopilado durante los meses de agosto a septiembre de 2016. En la segunda generación (D), nacidos entre 1946-1960 fueron entrevistadas tres personas, entre ellas, Edith Torres (1953). En la tercera generación (E), 1961-1975, escuchamos a cinco y la representamos con Omar Pescoso (1973). En la cuarta generación (F) 1976-1990, se entrevistaron a cuatro personas, de las cuales hace parte Eduardo Flores (1985) y en la quinta y última generación de estudio (G) 1991-2005, entrevistamos a seis jóvenes que hacen vida en Catia, entre ellos, a Ernesto La Cruz (1996).

La decisión de quién representaba sus generaciones se hizo con base en aquellos donde el testimonio lograba abarcar todos los aspectos de su propia experiencia vital y así entender el papel que representan dentro de la comunidad. En muchas ocasiones, lo que contaron se entrecruzaba con historias contadas desde otras generaciones. Por ejemplo, Ernesto La Cruz narró cómo se construyó el mercado de Catia ubicado en la plaza Peréz Bonalde, a través de las historias que sus abuelos le supieron contar:

[...] ]eso fue increíble para Catia porque esto no era un mercado. Toda Nueva Caracas estaba compuesta por granjas. En sí, en Catia estaban instaladas granjas, era una localidad campestre y rural. De hecho, te aseguro que aún en algunos lugares de Pérez Bonalde, existen granjas detrás de los edificios (Ernesto La Cruz).

Conocer esta historia a través de un joven de 20 años que no vivió cambios ni transformaciones de lo rural a lo urbano muestra que transmitir memorias de generación en generación es posible. Puede que ese relato no sea cierto pero para Ernesto La Cruz sus abuelos le dijeron una verdad sobre su comunidad.

Vale la pena resaltar que para la realización de las entrevistas, solicitamos a los interlocutores que se ubicaran en las generaciones que correspondía a su año de nacimiento a partir de la serie generacional precursora:

a. Generaciones 1901-2028, a partir de la serie generacional precursora

\footnotetext{
${ }^{24}$ De las generaciones identificadas por Viso (2012), nuestro estudio parte desde 1938-1998, por lo tanto, son decisivos para el análisis las generaciones que van desde los nacidos en las "zonas de fecha" 19311945 (C), 1946-1960 (D), 1961-1975 (E), 1976-1990 (F) y 1991-2005 (G).
} 


\begin{tabular}{|ccccccc|}
\hline Generaciones & Inicio & $\begin{array}{c}\text { GENERACIONES 1901-2028 } \\
\text { Intermedia }\end{array}$ & Final & $\begin{array}{c}\mathbf{\pm 3 0} \\
\text { años }\end{array}$ & $\begin{array}{c}\text { 士 Edad } \\
\text { en 2016 }\end{array}$ & $\begin{array}{c}\text { Aproximado } \\
\text { en 2016 }\end{array}$ \\
\hline A & 1901 & 1908 & 1915 & 1938 & $\dagger$ & $\dagger$ \\
\hline B & 1916 & 1923 & 1930 & 1953 & 93 & $(86-100)$ \\
\hline C & 1931 & 1938 & 1945 & 1968 & 78 & $(71-85)$ \\
\hline D & 1946 & 1953 & 1960 & 1983 & 63 & $(56-70)$ \\
\hline E & 1961 & 1968 & 1975 & 1998 & 48 & $(41-55)$ \\
\hline F & 1976 & 1983 & 1990 & 2013 & 33 & $(26-40)$ \\
\hline G & 1991 & 1998 & 2005 & 2028 & 18 & $(11-25)$ \\
\hline H & 2006 & 2013 & 2028 & 2043 & 3 & $(10-?)$ \\
\hline
\end{tabular}

Fuente: Elaboración a partir de serie generacional elaborada por Viso $(2012)^{25}$.

También utilizamos las fechas correspondientes a los periodos presidenciales de Venezuela desde 1930 hasta el presente según las generaciones del cuadro anterior, para estimular la memoria social histórica y presentamos una tercera tabla con las fechas que caracterizan la temporalidad de cada generación titulada "fases de la vida en la serie de generaciones de quince en quince años":

b. Fases de la vida en la serie de generaciones de quince en quince años.

\footnotetext{
${ }^{25}$ Estos datos son calculados a partir de la fecha intermedia de la Generación Precursora, por lo tanto, varían entre más o menos ( \pm ) siete años. Se le suma a la fecha intermedia, 15 años para infancia/adolescencia, 30 años al período de formación, 45 años al periodo de Iniciación de gestación, 60 años para el predominio de la gestión de dicha generación y 75 años para el periodo de salida, en base a la esperanza de vida del venezolano para el 2016.
} 


\begin{tabular}{|c|c|c|c|c|c|}
\hline Generaciones & $\begin{array}{c}\text { Infancia y } \\
\text { adolescencia }\end{array}$ & $\begin{array}{l}\text { Período de } \\
\text { formación }\end{array}$ & $\begin{array}{l}\text { Iniciación - } \\
\text { gestación }\end{array}$ & $\begin{array}{c}\text { Predominio } \\
\text { de gestión }\end{array}$ & $\begin{array}{l}\text { Posible } \\
\text { salida } i ?\end{array}$ \\
\hline & $0-15$ & $15-30$ & $30-45$ & $45-60$ & $60-75$ \\
\hline A & 1908-1923 & 1923-1938 & $1938-1953$ & $1953-1968$ & $1968-1983$ \\
\hline B & 1923-1938 & $1938-1953$ & $1953-1968$ & $1968-1983$ & 1983-1998 \\
\hline C & 1938-1953 & $1953-1968$ & $1968-1983$ & 1983-1998 & 1998-201: \\
\hline D & 1953-1968 & $1968-1983$ & 1983-1998 & $1998-2013$ & 2013-2028 \\
\hline E & 1968-1983 & $1983-1998$ & $1998-2013$ & $2013-2028$ & 2028-2043 \\
\hline $\mathrm{F}$ & 1983-1998 & $1998-2013$ & $2013-2028$ & $2028-2043$ & $2043-2058$ \\
\hline G & 1998-2013 & 2013-2028 & $2028-2043$ & $2043-2058$ & $2058-2073$ \\
\hline $\mathrm{H}$ & $2013-2028$ & $2028-2043$ & $2043-2050$ & $2058-2073$ & $2073-2088$ \\
\hline
\end{tabular}

Fuente: Elaboración a partir de serie generacional elaborada por Viso (2012).

Haciendo memoria, los interlocutores se ubicaron en sus generaciones y visualizaron la "zona de fechas" correspondiente a los períodos de vida, de esta forma, regresaron a sus marcos sociales para ubicar y construir por medio del lenguaje las relaciones sociales, espacios compartidos y experiencias de vida en Nueva Caracas.

\section{Conclusiones}

Este estudio sobre Nueva Caracas nos trajo elementos novedosos; primeramente, la cualificación de la memoria a través de las generaciones por medio de sustentos teóricos y metodológicos. También, la posibilidad de separar el paradigma de que la edad es únicamente cronológica para comprender la situación de vida que permite la convivencia entre coetáneos y contemporáneos, donde se generan criterios que relacionan a los participantes pertenecientes a distintas ' 'edades sociales". Además, logramos el rescate de la memoria colectiva de Catia encajada dentro de la urgencia de micro estudios urbanos para enfrentar los problemas comunitarios actuales. Elementos que nos permitieron contribuir en la reconstrucción de la memoria como acción social de Nueva Caracas-Catia, a través de las generaciones de 1938, 1953, 1968, 1983 y 1998, coetáneas y contemporáneas en 2016.

Podemos decir que mientras el pasado constituye la ruta de acontecimientos experimentados física e imaginariamente, es en el diseño que hacemos de nuestro futuro donde aparece la "novedad" y lo inesperado, junto con circunstancias que colocan a prueba a las personas manteniéndolos siempre alertas: el futuro es completamente intangible e indeterminable. En este sentido, cuando pedimos a Celina Fehr que nos cuente cómo se siente respecto a Nueva Caracas en la actualidad, aparece ese inesperado hecho que la coloca en "alerta":

Esta es la peor zona de Catia. Todo esto se echó a perder por falta de gobierno y cada vez estamos peor[...] hubo un cambio radical en Catia, un cambio profundo y triste[...] se han construido muchos edificios y muchas casas han sido derribadas, espacios que ya no se recuerdan y donde ya no hay espacios recreacionales ni familiares porque los comercios arrasaron con ellos. Además, la delincuencia ha hecho que muchas personas prefieran irse de Catia a permanecer acá (Celina Fehr). 
Es así como podemos visualizar la teoría de las generaciones, precisamente, por su componente metódico pues se nos hizo posible entrecruzar pasado e historia, así como también, el presente: desde Celina con 83 años de edad hasta Ernesto con 20 años de edad en 2016. Lo que nos permitió encontrar en el presente una misma realidad pero con temporalidades sociales distintas. Además, pudimos entrecruzar la memoria urbana con la idea de las generaciones encontrando el valor que puede tener el recordar espacios urbanos para quienes los habitan sobre todo en el aspecto sentimental y emocional. Por lo tanto, pensar la memoria de Nueva Caracas en sincronía con la historia de Caracas, y la historia propia de los venezolanos, nos permite acercarnos a sus generaciones históricas y, finalmente, conocer la memoria colectiva, las relaciones sociales, los encuentros y los desencuentros.

Si pensamos en lo urbano y sus lugares, nos damos cuenta que estos espacios "tienen" memoria gracias al significado y el sentimiento que les proveemos. A propósito de lo anímico y lo emocional, aprovechamos para citar a José Luis Vethencourt en "La ciudad y el alma":

[...]las diversas aventuras del espíritu de nuestros antepasados o de las generaciones que nos precedieron y que pueden expresarse emotivamente a través de ciertos monumentos en memoria de personajes ilustres [...] contribuiría a mantener vivas nuestras propias memorias ya dramatizadas (Vethencourt 1978: 3).

Esto nos recuerda la similitud en los recuerdos sobre el pasado en la plaza Peréz Bonalde, por ejemplo, Omar Pescoso dice "en mi infancia yo volaba papagayo ${ }^{26}$ por toda la plaza, en aquella época cerrábamos las calles y se podía manejar bicicleta por todas las cuadras[...] podíamos jugar con una pelota y compartir (Omar Pescoso)", en diálogo Eduardo Flores también comenta: "cuando yo era niño, en el boulevard corría muchísima brisa y podía volar papagayo. Era fenomenal estar ahí" (Eduardo Flores).

No obstante, frente a la necesidad de humanizar los lugares que nuestra memoria frecuenta, observamos que varios de los testimonios reconocían cambios dentro del "sentir" de la localidad. La situación de anomia que enfrenta Caracas ocasiona un amargo sabor y extrañamiento entre sus habitantes. Vethencourt (1978), definió como "submundos urbanos" a esta problemática: una forma de vida oculta que ataca y niega los principios éticos de la sociedad. Estos submundos funcionan como un cáncer y viven en las sombras, no son partes de las metas de la vida urbana y necesitan urgente auto-contención.

Entre los relatos no hubo historia oral narrada que no mencionara la inseguridad sufrida en las calles. Ernesto La Cruz, uno de los que representa las últimas generaciones, decía "[...]me quiero ir de Venezuela porque no puedo vivir solo[...] no importa cuánto me esfuerce o trabaje, no voy a poder. La gente cada vez es más violenta y por otro lado, cada vez estamos más asustados" (Ernesto La Cruz). Existe una profunda necesidad de humanizar los espacios de la ciudad escondida de Cabrujas (Cabrujas 1990 en Socorro, 1994). La falta de reconocimiento del otro, la ausencia de solidaridad y la poca pertenencia social reflejan un fuerte decaimiento en lo público y una ciudad atrapada por la delincuencia y servicios mal administrados que reflejan la dañina 'psicología del 'sálvese quien pueda' " (Vethencourt 1978: 4).

\footnotetext{
${ }^{26}$ Forma de llamar a los cometas o barriletes.
} 
En este diálogo, encontramos que la esperanza puede hallarse en la "inteligencia creadora" de las sociedades, que comprende el hacer memoria. Las generaciones sociales actuales de Nueva Caracas, reconocen que viven en una sociedad en constante decaimiento social, económico y político. Aquellos que aún recuerdan cómo era Catia, bien sea por memorias propias o ajenas, añoran un antiguo sistema de vigencias que reconocen a través de la nostalgia y que actualmente pertenece a un pasado lejano. Desconocemos qué o cuáles historias se estarán re-escribiendo actualmente en Catia pero tenemos la fuerte convicción en afirmar que las localidades necesitan humanizarse desde sus bases, y, la memoria nos ayuda a dibujar lo que somos o creemos ser, qué hemos sido y a dónde queremos ir. La memoria de los catienses podría servir de impulso para la re-construcción de un futuro posible estando en compañía de los otros que acompañan y forman parte de esas generaciones coetáneas y contemporáneas.

\section{Referencias Bibliográficas}

\section{- Libros}

Abad, Héctor. (2006): El olvido que seremos. Bogotá, Colombia: Planeta.

Benedetti, Mario (1995): El olvido está lleno de memoria. Buenos Aires, Argentina: Sudamericana.

Caïs, J., Folguera, L., y Formoso, C. (2014): Investigación cualitativa longitudinal. Madrid: Colección Cuadernos Metodológicos, Núm. 52. Centro de Investigaciones Sociológicas.

De Sola, I. (1967): Contribución al Estudio de los Planos de Caracas. La ciudad y la Provincia 1567-1967. Caracas: Comité de obras culturales del cuatricentenario de Caracas.

González, S. (2005): La ciudad Venezolana. Una interpretación de su espacio y sentido en la convivencia nacional. Caracas, Venezuela: Fundación para la cultura urbana.

Halbwachs, Maurice (2004): La Memoria Colectiva. Título original: La mémoire collective. Presses Universitaires de France, París (1968). España: Prensas Universitarias de Zaragoza.

Halbwachs, Maurice (2004): Los Marcos Sociales de la Memoria. Título original: Les cadres sociaux de la mémoire (1994). París: Albin Michel. Coedición: Facultad de Ciencias Sociales de la Universidad de Concepción en Chile y Facultad de Ciencias Económicas y Sociales de la Universidad Central de Venezuela. Caracas: Anthropos.

Marías, J. (1989): Generaciones y constelaciones. Madrid: Alianza editorial, S.A.

Marina, J. (2010): Las culturas fracasadas. El talento y la estupidez de las sociedades. Barcelona, España: Anagrama. Colección Argumentos. 
Martin, J.J. (2004): Diálogos reconstruidos para una historia de la Caracas moderna. Caracas: Universidad Central de Venezuela. Consejo de Desarrollo Científico y Humanístico.

Ortega y Gasset, J. (1957): El hombre y la gente. Obras inéditas. Madrid: Revista de Occidente.

Ortega y Gasset, J. (2005): En torno a Galileo. Madrid: Biblioteca Nueva.

Socorro, M. (1994): Catia Tres Voces. María Carrasquel Rivas, Henrique Hernández Mujica, José Ignacio Cabrujas”. Caracas: Fundarte.

Valery S.R. (1978): La Nomenclatura Caraqueña. Caracas, Venezuela: Ernesto Aristimuño.

Vázquez, F. (2001): La memoria como acción social. Relaciones, significados e imaginarios. Barcelona: Paidós Ibérica S.A.

\section{- Capítulos de libros}

Blanco, A. (1997): "Los afluentes del recuerdo: La memoria colectiva". En: J. M. RuizVargas (Comp.): Claves de la memoria (p. 83-106). Madrid, España: Trotta.

Cabrujas, José I. (1990): "La ciudad escondida", en: H. Seijas (comp.): Amada Caracas. Antología (esencial) de la ciudad contemporánea. Caracas: El perro y la rana, 2014: pp. 133-146.

Eco, Umberto (2010): "Personajes imaginarios y ciudades reales". En: T. Hernández (Comp.), Ciudad, espacio público y cultura urbana. 25 conferencias de la cátedra Permanente de Imágenes Urbanas. Traducciones y notas de Rocco Mangieri. Caracas: Fundación para la Cultura urbana, Grupo de Empresas Econoinvest.

Silva, A. (1994): "Algunos imaginarios urbanos desde centros históricos de América Latina”. En: Carrión F. (Ed.) La ciudad construida. Urbanismo en América Latina (2001). Ecuador: FLACSO.

\section{- Inéditas}

Viso, C. (2012): Cultivador de humanidad entre la niebla. Rafael Augusto Vegas Sánchez. (1908-1973). (Documento inédito) Capítulo: El programa de Febrero 1936: Sanear, Educar y Poblar: pp. 39-47.

\section{- Documentos en línea}

Durán, Guillermo: "Catia: Semblanza de una localidad caraqueña". Publicado por: Cronista de Caracas. Última edición: 07 de diciembre de 2016. Consultado por última vez el 04 de Enero de de 2021. Disponible en: https://www.facebook.com/notes/cronista-de-caracas/catia-semblanza-de-una-localidad-caraqueña/1188895937866111

Fundación Arquitectura y Ciudad: "1940, se drena y colmata la Laguna de Catia". Publicado el 12 de marzo de 2018. Consultado por última vez el 03 de Enero de 2021. Disponible en: https://fundaayc.wordpress.com/2018/03/12/1940•/ 
González, L. y Marín, O. (2009): "Caracas al mar: El acceso de Caracas al Litoral y el proyecto de túnel por Altamira (1948-1958)". Publicado por: Revista Tiempo y Espacio v.19 n.51. Caracas. Consultado por última vez: 05 de Enero de 2021. Disponible en: http://ve.scielo.org/scielo.php?pid=S1315-94962009000100002\&script=sci_abstract

Provenzali, Lepoldo. (Agosto de 2012): "Irrupciones en la memoria de la ciudad". Nota periodística Tal Cual. Consultado por última vez el 14 de Enero de 2021. Disponible en: http://asmenlinea.blogspot.com/2012/08/irrupciones-en-la-memoria-de-la- ciudad.html.

\section{- Blogs}

Procatia. "Hacia la construcción de Catia Municipio". Portal web de la organización. Consultado por última vez el 02 de Enero de 2021. Disponible en: https://procatia.wordpress.com.

\section{- Youtube}

Iconos de Caracas: El Torero, una ventana al pasado" (Vídeo en YouTube). Publicado el 19 de agosto de 2013 por "Tu Zona Caracas". Disponible en: https://www.youtube.com/watch?v=D1fVCC7zMGQ (Consultado por última vez el 20 de enero de 2021).

\section{- Trabajos de grado}

Allen, A.J.: Los medios de comunicación impresos y la reconstrucción de la memoria colectiva. Período 1999-2006. Caracas, Universidad Central de Venezuela, Facultad de Ciencias Económicas y Sociales, Escuela de Sociología, 2006. (Trabajo Final de Grado para optar por el Título de Socióloga).

Antillano, J.G.: Catia: 1845-1986 (Estudio de Historia Local). Caracas, Universidad Central de Venezuela. Facultad de Humanidades y Educación, Escuela de Historia, 1999. (Monografía presentada para optar por el Título de Licenciado en Historia).

Escobar, R.: Memoria Histórica e Identidad (Escuela Taller La Guaira como estrategia educativa para la defensa, protección, cuidado y conservación del Patrimonio Cultural Arquitectónico del Casco Colonial de La Guaira). Caracas, Universidad Central de Venezuela, Escuela de Sociología, 2012. (Trabajo Final de Grado para optar al título de Socióloga)

Granados, R. (2015): El consejo de preservación y desarrollo (COPRED) en la ejecución de estrategias educativas en la Ciudad Universitaria de Caracas. Caracas, Universidad Central de Venezuela, Facultad de Humanidades y Educación, Escuela de Educación, 2015. (Trabajo Final de Grado para optar a la Licenciatura en Educación, Mención Diseño y Gestión de Proyectos Educativos)

\section{- Conferencias}

Provenzali, L. (2006): "Un sueño para Catia. De Parroquia a Municipio". Conferencia dictada en ProCatia y G. Ferrero. Presentación en colaboración con Liderazgo y Visión, SPUGA-ADMC. Caracas, Venezuela. 
Vethencourt, José Luis. (1978): "La Ciudad y el Alma". Conferencia dictada en FAUUCV., con motivo del primer seminario sobre la ciudad. Instituto de Arquitectura y Urbanismo (I.A.U). Archivo recuperado del I.A.U. Caracas, Venezuela.

\section{- Documentales}

Dalsgaard, A. (productor y director). 2012. The human Scale. Documental. Dinamarca.

De Pedro, M. (guión y dirección). 1975. Juan Vicente Gómez y su época. [Documental]. Venezuela.

Lucien, O. (guión y dirección). 2000. El espacio interior de Carlos Raúl Villanueva. [Documental]. Venezuela.

Oteyza, C. y Garmendia S. (guión y dirección). 1997. El General López Contreras, la transición. [Documental]. Venezuela.

Oteyza, C. y Garmendia S. (guión y dirección). 1992. Isaías Medina Angarita, soldado de la libertad. [Documental]. Venezuela. 